\title{
Transient Boundary Element Method and Numerical Evaluation of Retarded Potentials
}

\author{
Ernst P. Stephan ${ }^{1}$, Matthias Maischak ${ }^{2}$, and Elke Ostermann ${ }^{1}$ \\ ${ }^{1}$ Leibniz Universität Hannover, Institut für Angewandte Mathematik, \\ Am Welfengarten 1, 30167 Hannover, Germany \\ \{stephan, osterman\}@ifam.uni-hannover.de \\ ${ }^{2}$ Brunel University, School of Information Systems, Computing \& Mathematics \\ John Crank Building, Uxbridge UB8 3PH, United Kingdom \\ matthias.maischak@brunel.ac.uk
}

\begin{abstract}
We discuss the modeling of transient wave propagation with the boundary element method (BEM) in three dimensions. The special structure of the fundamental solution of the wave equation leads to a close interaction of space and time variables in a so-called retarded timeargument. We give a detailed derivation of the discretization scheme and analyse a new kind of "geometrical light cone" singularity of the retarded potential function. Moreover, we present numerical experiments that show these singularities.
\end{abstract}

Keywords: retarded potential, transient boundary element method.

\section{Introduction}

The simulation of sound radiation for automotive systems has become of great industrial interest and here the transient boundary element method is a powerful tool, especially for high frequency radiation phenomena. Nevertheless, the simulation of three dimensional time dependent problems is a challenging task. HaDuong 1] was able to prove the unconditional stability of the transient boundary element method with Galerkin's method in space and time. Hence, the question arises, why have so many instabilities in numerical experiments been reported. For a first kind integral equation with the retarded single layer potential we perform a p-version boundary element method both in space and time. We address especially the question of how to gain an accurate quadrature method. Thus we analyse the potentials that arise in the computation of the Galerkin elements and show that a new kind of "geometrical light cone" singularity can cause severe inaccuracies in the computation of the Galerkin stiffness matrix. Therefore, an accurate quadrature scheme which respects these singularities, e.g. by an appropriate grading, needs to be used for the computation of the matrix entries.

The paper is structured as follows. First, we represent the solution $u$ of the Dirichlet problem of the wave equation via a single layer ansatz with unknown density function $p$ and derive a first kind integral equation for $p$ on the surface $\Gamma$ of the scatterer. For this integral equation we give a variational formulation which 
we solve approximately with Galerkin's method using piecewise polynomials in space and time.

We show in detail how the retarded time argument in the kernel function of the single layer potential influences the entries of the Galerkin stiffness matrix. Here the time discretization leads to special "domains of influence" $E_{k}$, annular regions in space whose radii are given by the time discretization. If the triangle of the trial function and $E_{k}$ do not overlap, the resulting matrix entry vanishes. For the computation of these entries, we introduce a new quadrature scheme paying special attention to the interaction between the triangles and the domain of influence. We show that the retarded integral operators poss "geometrical light cone" singularities (section 4). Finally, we present numerical experiments in $\mathbb{R}^{3}$ confirming this fact.

\section{Model Problem and Single Layer Potential}

Let us consider the transient sound radiation of some bounded domain $\Omega^{-}$, a bounded open domain with connected complement $\Omega:=\mathbb{R}^{3} \backslash \bar{\Omega}^{-}$. We investigate the wave equation for the displacement $u(t, x)$ with $t \in \mathbb{R}_{+}, x \in \Omega$

$$
\frac{\partial^{2} u}{\partial t^{2}}-\Delta u=0
$$

We assume causal functions $f(t)$, i.e. $f(t<0)=0$ and use the initial conditions

$$
u(0, x)=u_{t}(0, x)=0 \quad \text { for } x \in \Omega,
$$

where the lower index $t$ indicates the time derivative. Given Dirichlet boundary conditions on $\Gamma:=\partial \Omega$

$$
u=f \quad \text { on } \mathbb{R} \times \Gamma .
$$

We make a single layer potential ansatz for the solution $u$

$$
u(t, x)=S p(t, x)=\frac{1}{4 \pi} \int_{\Gamma} \frac{p(t-|x-y|), y}{|x-y|} d s_{y} \quad(x \notin \Gamma),
$$

with density function $p$ and the retarded time argument $t-|x-y|$. The latter connects the space and time variables and physically results in the retarded signal of a source point. As we will see later, this retarded time argument has a significant impact on our discretization scheme. As the single layer potential $S$ is continuous everywhere, we have for its limit on $\Gamma$

$$
V p(t, x)=\frac{1}{4 \pi} \int_{\Gamma} \frac{p(t-|x-y|), y}{|x-y|} d s_{y} \quad(x \in \Gamma) .
$$

and using (3), there holds the boundary integral equation for $p$

$$
f(t, x)=V p(t, x) .
$$


Note that the mapping $V$ is continuous (see [1]). We can deduce the following variational formulation: given $f$, find $p$ such that for all $\eta$

$$
\int_{0}^{\infty} \int_{\Gamma} V p(t, x) \eta(t, x) d s_{x} d t=\int_{0}^{\infty} \int_{\Gamma} f(t, x) \eta(t, x) d s_{x} d t
$$

Due to the theory of Ha-Duong [1] this formulation is uniquely solvable in appropriate Sobolev spaces on $\Gamma$ and $[0, \infty)$. It can be solved approximately with a Galerkin scheme in space and time for appropriate piecewise polynomial subspaces. For some convergence results see 1 and the references therein. We concentrate on suitable quadrature schemes for actually computing the Galerkin solution leading to a fully discrete system discussed in the next section.

\section{The Discrete Problem}

In the following we denote by $N_{t}$ the number of degrees of freedom in time and by $N_{s}$ the number of degrees of freedom in space. Let us assume, that the boundary $\Gamma$ is subdivided into triangles $T_{j}$ with maximal diameter $h$ and let us assume that we have a decomposition of the time interval $[0, T]$ with $T<\infty$, into $N_{t}$ subintervals with step size $\Delta t$. Let $t_{n}=n \Delta t$.

We discretize the variational form of the boundary integral equation (17) using basis functions of order at least constant in both space and time, such that

$$
p_{h}(t, x)=\sum_{m=1}^{N_{t}} \sum_{i=1}^{N_{s}} b_{i}^{m} \gamma^{m}(t) \varphi_{i}(x)=\sum_{m=1}^{N_{t}} \gamma^{m}(t) \phi^{m}, \gamma^{m} \in S^{p_{t}}(\Delta t), \varphi_{i} \in S^{p_{s}}(h)
$$

where $\gamma^{m}(t)$ is a Legendre polynomial of degree $p_{t}$

$$
\gamma^{m}(t)= \begin{cases}\sum_{i=0}^{p_{t}} \beta_{i} t^{i} & \text { for } t \in\left(t_{m}, t_{m+1}\right] \\ 0 & \text { else }\end{cases}
$$

Then, the p-version of the Galerkin scheme reads: find $p_{h}$ as in (8), such that

$$
\int_{0}^{T} \int_{\Gamma} V p_{h}(t, x) \gamma^{n}(t) \varphi_{j}(x) d s_{x} d t=\int_{0}^{T} \int_{\Gamma} f(t, x) \gamma^{n}(t) \varphi_{j}(x) d s_{x} d t
$$

for $n=1, \ldots, N_{t}$ and $j=1, \ldots, N_{s}$. For the left hand side, we have to compute integrals of the type

$$
V_{i j m n}:=\frac{1}{4 \pi} \int_{0}^{T} \int_{\Gamma} \int_{\Gamma} \frac{1}{|x-y|} \gamma^{m}(t-|x-y|) \varphi_{i}(y) \gamma^{n}(t) \varphi_{j}(x) d s_{y} d s_{x} d t
$$

If we change the order of integration, we can evaluate the time integral analytically and the resulting integral only depends on the time difference, such that $V_{i j m n}=V_{i j}^{n-m}$. Let $k:=n-m$ and let $p \in \mathbb{N}$, then 


$$
\int_{0}^{T} t^{p} \mathbf{1}_{t-|x-y| \in\left(t_{0}, t_{1}\right]} \mathbf{1}_{t \in\left(t_{k}, t_{k+1}\right]} d t= \begin{cases}\frac{1}{p+1}\left(\left(t_{1}+|x-y|\right)^{p+1}-t_{k}^{p+1}\right) & (x, y) \in E_{k-1} \\ \frac{1}{p+1}\left(t_{k+1}^{p+1}-|x-y|^{p+1}\right) & (x, y) \in E_{k} \\ 0 & \text { else }\end{cases}
$$

where 1 denotes an indicator function on the time intervals and we define the domain of influence (light cone) $E_{k}$ by

$$
E_{k}:=\left\{(x, y) \in \Gamma \times \Gamma: t_{k} \leq|x-y| \leq t_{k+1}\right\} .
$$

The rather unusual domain $E_{k}$ is due to the retarded time argument in the single layer potential.

Let us now return to the evaluation of the Galerkin integrals (10). As the matrix depends only on the time difference, we can translate our basis functions $\gamma^{m}(t)$ and $\gamma^{n}(t)$ in time onto $\left(t_{0}, t_{1}\right)$ and $\left(t_{k}, t_{k+1}\right)$, respectively. To simplify the notation, we indicate all terms connected to the ansatz function $\gamma^{m}(t)$ with the index $A$ and for the test function $\gamma^{n}(t)$ with $T$, thus $\gamma^{m}(t)$ is of polynomial degree $p_{A}$ and $\gamma^{n}(t)$ of degree $p_{T}$. Using (11), applying the binomial series and reordering, we can rewrite (10) with $k:=n-m$

$$
\begin{aligned}
V_{i j}^{k}= & \sum_{l=0}^{p} \alpha_{l}^{k-1} \iint_{E_{k-1}}|x-y|^{l-1} \varphi_{i}(x) \varphi_{j}(y) d s_{x} d s_{y} \\
& +\sum_{l=0}^{p} \alpha_{l}^{k} \iint_{E_{k}}|x-y|^{l-1} \varphi_{i}(x) \varphi_{j}(y) d s_{x} d s_{y}
\end{aligned}
$$

where

$$
\begin{aligned}
& \alpha_{l}^{k}:=\left\{\begin{array}{cl}
\sum_{i=0}^{p_{A}} \sum_{j=0}^{p_{T}} \frac{\beta_{i}^{A} \beta_{j}^{T}}{i+j+1} t_{k+1}^{i+j+1} & l=0 \\
\sum_{j=0}^{p_{T}} \beta_{j}^{A}\left(\sum_{i=l}^{p_{m}} \beta_{i}^{T} \frac{(-1)^{l}}{i+j-l+1}\left(\begin{array}{l}
i \\
l
\end{array}\right) t_{k+1}^{i+j-l+1}\right. & \\
\left.\quad+\beta_{l-j-1}^{A} \sum_{l=0}^{l-j-1}\left(\begin{array}{c}
l-j-1 \\
d
\end{array}\right) \frac{(-1)^{d+1}}{l-d}\right) & l=1, \ldots, p_{A} \\
\sum_{j=0}^{p_{T}} \beta_{j}^{T} \beta_{l-j-1}^{A} \sum_{l=0}^{l-j-1}\left(\begin{array}{l}
l-j-1 \\
d
\end{array}\right) \frac{(-1)^{d+1}}{l-d} & l=p_{A}+1, \ldots, p_{A}+p_{T}+1
\end{array}\right. \\
& \alpha_{l}^{k-1}:=\left\{\begin{array}{l}
\sum_{i=l}^{p_{A}} \sum_{j=0}^{p_{T}} \beta_{i}^{A} \beta_{j}^{T}\left(\begin{array}{l}
i \\
l
\end{array}\right) \frac{(-1)^{l}}{i+j-l+1} t_{k}^{i+j-l+1} \\
\quad+\sum_{j=0}^{p_{T}} \beta_{l-j-1}^{A} \beta_{j}^{T} \sum_{f=0}^{l} \sum_{d=0}^{\min (l-j-1, f)}(-1)^{l}\left(\begin{array}{c}
l-j-1 \\
d
\end{array}\right)\left(\begin{array}{l}
l-d \\
f-d
\end{array}\right) \quad l=0, \ldots, p_{A} \\
\sum_{j=0}^{p_{T}} \beta_{l-j-1}^{A} \beta_{j}^{T} \sum_{f=0}^{l} \sum_{d=0}^{\min (l-j-1, f)}(-1)^{l}\left(\begin{array}{c}
l-j-1 \\
d
\end{array}\right)\left(\begin{array}{c}
l-d \\
f-d
\end{array}\right) \quad l=p_{m}+1, \ldots, p_{A}+p_{T}+1
\end{array}\right.
\end{aligned}
$$

We see from (13) that the entries of the Galerkin matrix are given by a sum of integrals with integration domains depending on the time step light cone and 
with kernels only depending on the space variables. Therefore, returning to (9), we can rewrite our Galerkin scheme with (8) as the linear system

$$
\sum_{m=1}^{n} V^{n-m} \phi^{m}=F^{n} \quad\left(n=1, \ldots, N_{t}\right),
$$

where the matrix entries are defined by (13).

We can formulate a time stepping algorithm for (14) as summarized in the time stepping algorithm. In each time step we have to compute a new matrix $V^{n-m}$ and a new solution vector $\phi^{n}$. The matrices are sparsely populated due to the restriction of the interaction of all triangles with the domain of influence and, as the radii are time dependent and our body $\Omega^{-}$is bounded, at some point the domain of influence has passed the body and the new matrices vanish. Note that the system matrix $V^{0}$ remains the same in each time step. Also note that $F^{n}$ is computed via numerical evaluation of the right hand side of (9).

As mentioned before, for the above algorithm severe numerical instabilities have been reported, although Ha-Duong proved its unconditional stability [1]. In the next section we point out special "geometrical light cone" singularities, that occur in the computation of the inner integral of the integrals given in (13), which have to be dealt with through the development of a sufficiently accurate quadrature scheme for the outer integral in (13) in order to achieve a sufficiently accurate quadrature for the integral.

\section{Time Stepping Algorithm.}

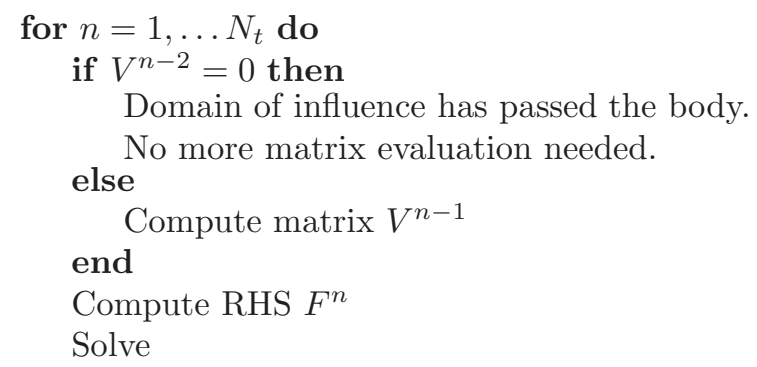

$$
V^{0} \phi^{n}=F^{n}-\sum_{m=1}^{n-1} V^{n-m} \phi^{m}
$$

and store new solution vector $\phi^{n}$. end

\section{Analysis of the Retarded Potential and Quadrature Scheme}

In this section we analyse the behavior of typical potential terms like (15) below. Such an integral has a different smoothness depending on whether the light cone 
hits a corner or an edge of the triangle $T$, see the situations given in Fig. 4. As we see in the proof of Theorem 1 the solution of the potential becomes singular in the second case.

In the following we restrict ourselves to constant basis functions in space and time and use a numerical quadrature for the outer integrals in (13). Let us take $y \in \mathbb{R}^{3}$ as a quadrature point. Then, when the integration is performed only on one triangle $T \in \mathbb{R}^{3}$, the integration domain becomes

$$
E_{T}:=\left\{x \in T: r_{\min } \leq|x-y| \leq r_{\max }\right\} \quad y \in \mathbb{R}^{3} \text { (fixed), }
$$

with $0 \leq r_{\min }<r_{\max }$. Thus the integrals are of the type

$$
I(y)=\int_{E_{T}} k(x, y) d s_{x},
$$

where $k(x, y)=|x-y|^{\alpha}$ for $\alpha \geq-1$. In the following, we will refer to (15) as the potential integral.

Let us focus on the situation where the triangle $T$ and the quadrature point $y$ lie in the same plane. Introduce a local coordinate system with respect to the triangle $T$. The domain of influence of the triangle $E_{T}$ becomes for fixed $y \in \mathbb{R}^{3}$ an annular ring. Therefore, we introduce polar coordinates $(r, \varphi)$ in our local coordinate system with origin $y$, orientate our triangle under these settings and devide the triangle into elements of the type as sketched in Fig. 11 The upper
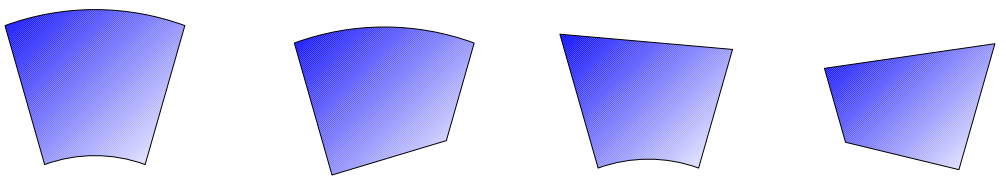

Fig. 1. 4 Quadrature Elements

and lower boundaries of all these elements can be parameterized as $r(\varphi)$ and hence the integrals (15) can be computed (both analytically and numerically).

The generalization to the situation where $y$ and $T$ do not lie in the same plane can be solved by applying the above scheme, as the kernel $k(x, y)$ only depends on the distance between $x$ and $y$, see Fig. 2 (a more detailed description of the quadrature scheme is given in 2/3]). The following theorem describes the regularity of the typical potentials (15) and gives the most relevant singularities resulting from so-called edge-singularities. When taking higher derivatives of the potentials even more singularities might appear, but this is not further investigated here for brevity.

Theorem 1. The potential integrals (15), i.e. I $\epsilon$ ) in (16) and (17), posses "geometrical light cone" singularities, which are of order $\epsilon^{-1 / 2} \ln \epsilon$ for the case $\alpha=-1$. These singularities lie parallel to the triangle sides with distances $r_{\min }$ and $r_{\max }$ as illustrated in Fig. 3 . 
Proof. In order to understand, what is happening when the light cone hits the triangle we study the following two reference situations. Here we choose a ball of fixed radius $R$ with center $m_{\epsilon}(\epsilon \geq 0)$ moving either towards an edge or a corner. Note that $R$ corresponds to $r_{\min }$ and $r_{\max }$. These two reference situations are sketched in Fig. 4 ,

For the corner, the reference situation (Fig. [4 left) is as follows. We have two rays $g_{1}$ and $g_{2}$ given by $\gamma\left(\begin{array}{l}0 \\ 1\end{array}\right)$ and $\gamma\left(\begin{array}{l}1 \\ 0\end{array}\right)$ respectively, with $\gamma \geq 0$. We move the center of the ball along $\left(\begin{array}{l}1 \\ 1\end{array}\right)$ towards these rays with $m_{\epsilon}=\frac{\epsilon-R}{\sqrt{2}}\left(\begin{array}{l}1 \\ 1\end{array}\right)(\epsilon \geq 0)$. Thus the intersection points with the rays are

$$
\begin{aligned}
& S_{1}(\epsilon)=\left(0, \frac{1}{\sqrt{2}}\left((\epsilon-R)+\sqrt{2 R^{2}-(\epsilon-R)^{2}}\right)\right)^{T} \\
& S_{2}(\epsilon)=\left(\frac{1}{\sqrt{2}}\left((\epsilon-R)+\sqrt{2 R^{2}-(\epsilon-R)^{2}}\right), 0\right)^{T}
\end{aligned}
$$

For our integral (15) we have $I(y)=I\left(m_{\epsilon}\right)$ and therefore

$$
\begin{aligned}
I\left(m_{\epsilon}\right)=: I(\epsilon) & =\int_{0}^{\frac{\pi}{2}} \int_{0}^{\frac{1}{\sqrt{2}}\left((\epsilon-R)+\sqrt{2 R^{2}-(\epsilon-R)^{2}}\right)} d r d \varphi \\
& =\frac{\pi}{2 \sqrt{2}}\left((\epsilon-R)+\sqrt{2 R^{2}-(\epsilon-R)^{2}}\right)
\end{aligned}
$$

thus

$$
I^{\prime}(\epsilon)=\frac{\pi}{2 \sqrt{2}}\left(1+\frac{R-\epsilon}{\sqrt{2 R^{2}-(\epsilon-R)^{2}}}\right)
$$

We see that $I^{\prime}(\epsilon) \rightarrow \frac{\pi}{\sqrt{2}}$ for $\epsilon \rightarrow 0$. The singularity for $\epsilon \rightarrow(1+\sqrt{2}) R$ is only relevant if the circle lies completely in the triangle.

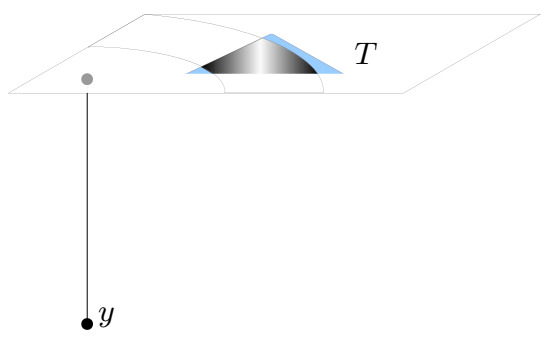

Fig. 2. Geometry of quadrature

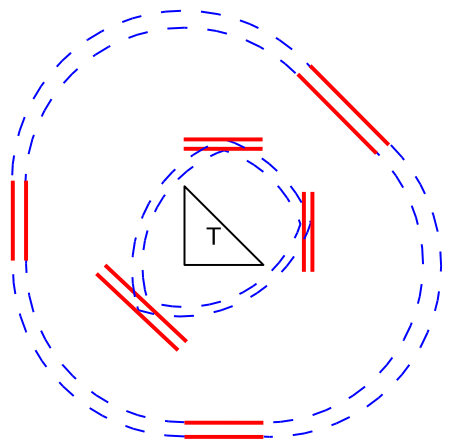

Fig. 3. Triangle light cone with "geometrical light cone" singularities indicated by 12 straight line segments 

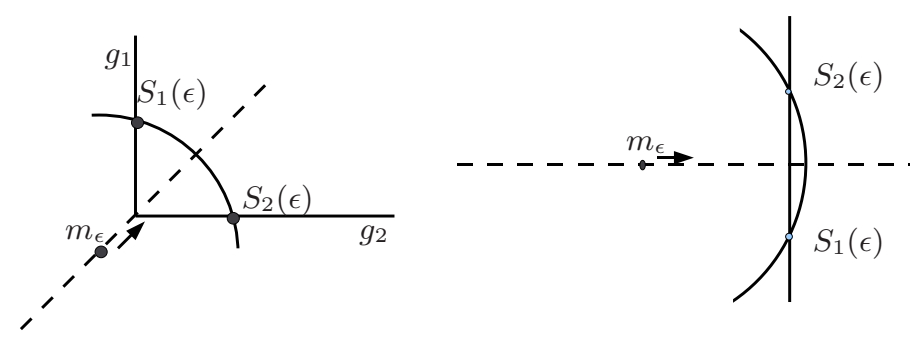

Fig. 4. Corner reference situation (left) and edge reference situation (right)

In order to understand, what happens when a sphere meets the edge of a triangle (see Fig. 4 , right), we choose a reference edge along $\gamma\left(\begin{array}{l}0 \\ 1\end{array}\right)(\gamma \in \mathbb{R})$ and a sphere with midpoint $\left(-R, \frac{1}{2}\right)^{T}$. This sphere intersects with the edge at the points $S_{1}(\epsilon)=\left(0, \frac{1}{2}+\sqrt{\epsilon(2 R-\epsilon)}\right)^{T}$ and $S_{1}(\epsilon)=\left(0, \frac{1}{2}-\sqrt{\epsilon(2 R-\epsilon)}\right)^{T}$, when the midpoint is moving towards the edge $m_{\epsilon}=\left(\epsilon-R, \frac{1}{2}\right)^{T}$, where $\epsilon \geq 0$. The angle of $S_{1}(\epsilon)$ relative to $m_{\epsilon}$ is now

$$
\varphi_{1}=\arctan \left(\frac{\sqrt{\epsilon(2 R-\epsilon)}}{\epsilon-R}\right)
$$

and the angle of $S_{2}(\epsilon)$ is $\varphi_{2}=2 \pi-\varphi_{1}$.

With $r_{1}(\varphi)=\frac{\sqrt{\epsilon(2 R-\epsilon)}}{\sin \varphi}$ and $r_{2}(\varphi)=R$, (15) becomes in polar coordinates

$$
\begin{aligned}
I(\epsilon) & :=\int_{-\varphi_{1}}^{\varphi_{1}} \int_{r_{1}(\varphi)}^{r_{2}(\varphi)} d r d \varphi \\
& =2 R \varphi_{1}-2 \sqrt{\epsilon(2 R-\epsilon)} \ln \tan \frac{\varphi_{1}}{2} \\
& =: I_{1}(\epsilon)+I_{2}(\epsilon) .
\end{aligned}
$$

Now, for \pm determined by the sign of $\cos \left(\varphi_{1}\right)$, it holds

$$
\begin{aligned}
I_{1}^{\prime}(\epsilon)= & \frac{2 R}{\sqrt{\epsilon(2 R-\epsilon)}} \\
I_{2}^{\prime}(\epsilon)= & \frac{2(R-\epsilon)}{\sqrt{\epsilon(2 R-\epsilon)}}\left(\ln \left(\frac{\sqrt{\epsilon(2 R-\epsilon)}}{(\epsilon-R)(1 \pm R(\epsilon-R))}\right)\right) \\
& \quad+\frac{2(R-\epsilon)}{\epsilon(2 R-\epsilon)}-\frac{2 \pm 4 R(\epsilon-R)}{(\epsilon-R)(1 \pm R(\epsilon-R))} .
\end{aligned}
$$

Therefore, the derivative of $I(\epsilon)$ becomes unbounded for $\epsilon \rightarrow 0, \epsilon \rightarrow R$ and $\epsilon \rightarrow 2 R$.

What does this mean for (15)? The singularity for $\epsilon=R$ corresponds to the case where $y$ lies on the edge of a triangle in (15), and this corresponds to the 

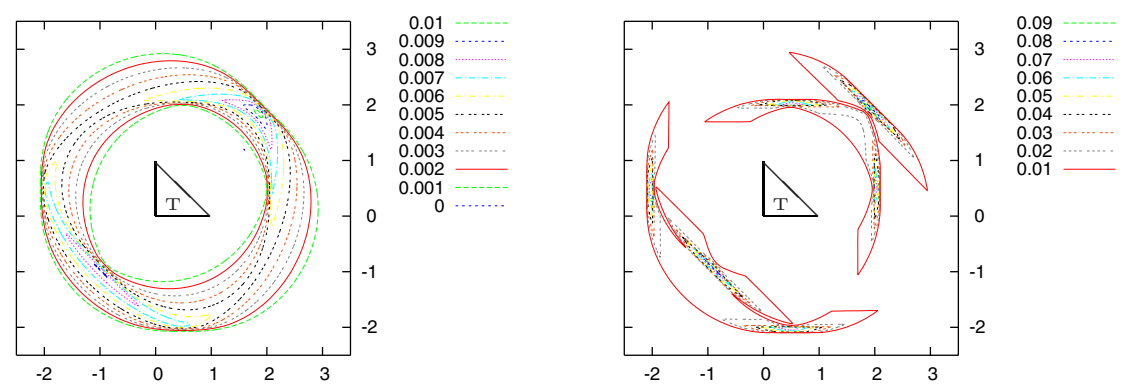

Fig. 5. Contour plot of the triangle-potential (left) and the norm of its gradient (right) for $r_{\min }=2.0, r_{\max }=2.1$

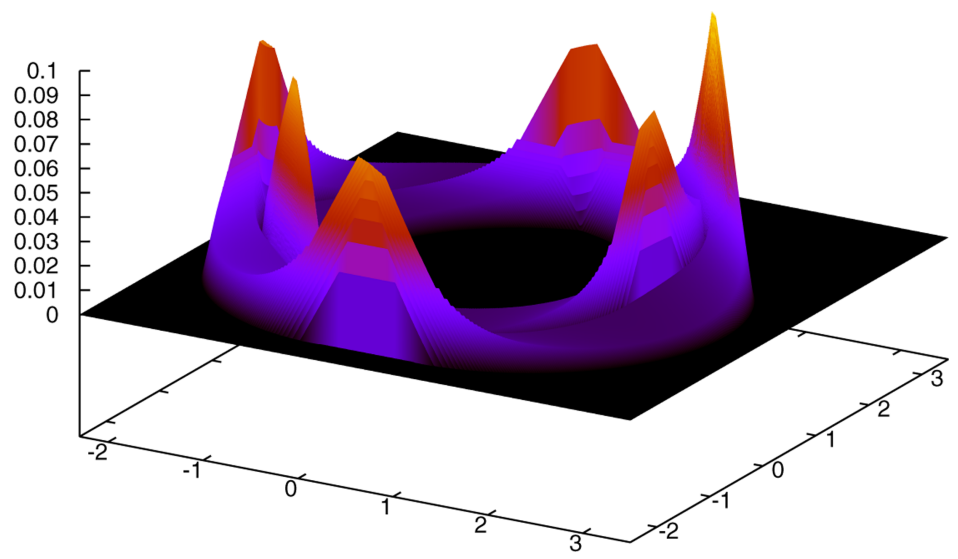

Fig. 6. Surface plot of the norm of the gradient of the triangle potential for $r_{\min }=$ $2.0, r_{\max }=2.1$

so called edge singularities for solutions of 3D boundary value problems, which are singular due to the kernel. For $\epsilon=0$ and $\epsilon=2 R$, we have geometrical singularities that are occurring when the boundary of the light cone $E_{T}$ passes the edge with the outer and inner circle with radius $r_{\max }$ and $r_{\min }$, respectively.

If we apply the polar quadrature scheme described above to the reference triangle with vertices $(0,0)^{T},(0,1)^{T},(1,0)^{T}$, we can observe exactly the behavior discussed in Theorem [1. In the left plot of Fig. 5 we see the contour plot of integral (15) with $k(x, y)=|x-y|^{-1}$. We fix the usually time dependent radii to $r_{\max }=2.1$ and $r_{\min }=2.0$ and evaluate (15) for $y \in[-2.5,3,5]^{2}$. We observe that the contour levels become dense at the propagated edges, see Fig. 3. This observation is underlined by the right plot of Fig. 5, where the norm of the gradient of (15) is plotted and the propagated edges become more visible. In Fig. 6 the gradient of the potential of the triangle is plotted again as a $3 \mathrm{D}$ plot. 
Let us finally remark, that the domain of influence of a triangle is of course a three dimensional domain. In this paper we have restricted our analysis to the case where a triangle $T$ and a point of observation $y$ are in the same plane, but the main arguments hold true for the fully three dimensional case. This will be a topic of a further paper. Here, our main result is that the classical near field and far field notation of the elliptic integral operators does not hold true for hyperbolic problems. Nevertheless, from our potential analysis (Thm. 1) we can obtain a grading strategy for the outer quadrature of the Galerkin integrals.

\section{References}

1. Ha-Duong, T.: On retarded potential boundary integral equations and their discretisation. In: Topics in computational wave propagation. Lect. Notes Comput. Sci. Eng, vol. 31, pp. 301-336. Springer, Berlin (2003)

2. Maischak, M., Ostermann, E., Stephan, E.P.: On the numerical evaluation of retarded potentials. Institut für Angewandte Mathematik, Leibniz Universität Hannover, Preprint no. 89 (2007)

3. Ostermann, E.: PhD thesis, Institut für Angewandte Mathematik, Leibniz Universität Hannover (in preparation) 